Epoca

Angulo Distancia No. Peso

$\Sigma 2745 . \quad$ Mag. 5.6, 7.7.

$\alpha=20^{\mathrm{h}} 5^{8 \mathrm{~m}} 8 \quad \delta=-6^{\circ} \mathrm{I}^{\prime}$.

1900.668

${ }_{1} 88^{\circ} 5^{6^{\prime}} \quad 2 ! 47 \quad 2 \quad 3$

Sensiblemente fija.

$\Sigma$ 2799. Mag. 6.6, 6.6.

$\alpha=21^{\mathrm{m}} 24^{\mathrm{m}} \circ \delta=+10^{\circ} 39^{\prime}$

I 900.79I I $\quad 10^{\circ} 0^{\prime} \quad$ I."2 I $\quad 2 \quad 3$

Muy sensible movimiento.

$\beta$ 1212. Mag. 6.3, 7.1.

$\alpha=2 \mathrm{I}^{\mathrm{h}} 34^{\mathrm{m}} \cdot 4 \quad \delta=-0^{\circ} 3 \mathrm{I}^{\prime}$.

$1900.668 \quad 205^{\circ} 5^{\prime}$ (est.) $0^{\prime \prime} 50 \quad 4 \quad 3$

Considero seguro el alargamiento, no obstante no pueden conciliarse mis observaciones con el ángulo dado por Mr. Aitken (A. N. 3584) $p=269^{\circ} \circ$, 1898.78 .

$$
\begin{aligned}
& \beta{ }_{276}=\eta \text { Piscis Austrini. Mag. 6.5, 7.0 } \\
& \alpha=2 \mathrm{i}^{\mathrm{h}} 55^{\mathrm{m}} \mathrm{t} \quad \delta=-28^{\circ} 5^{6^{\prime}} \text {. } \\
& \text { I900.769 I I I }{ }^{\circ} 8^{\prime} \quad \text { I"68 } 33
\end{aligned}
$$

Dos medidas de distancia. Fija.

$\beta 172=51$ Aquarii. Mag. 5.6, 5.7.

$$
\alpha=22^{\mathrm{h}}{ }^{2} 8^{\mathrm{m}} .9 \quad \delta=-5^{\circ} 20^{\prime} \text {. }
$$

\begin{tabular}{rrrrrr}
1900.654 & $356^{\circ} 7^{\prime}$ (est.) 0.6 & 3 & 2 \\
1900.663 & 8 & 82 & 0.6 & 4 & 3 \\
\hline 1900.66 & 424 & 0.6 &
\end{tabular}

Dificil, pero por momentos la desdoblo bien. Binaria.

$\mathrm{Hh}{ }_{762}=53$ Aquarii. Mag. 6.0, 6.o.

$$
\alpha=22^{\mathrm{h}} 2 \mathrm{1}^{\mathrm{m}} \cdot 2 \quad \delta=-\mathrm{I} 7^{\circ} \mathrm{I}^{\prime} \text {. }
$$

$\begin{array}{llllll}1900.668 & 308^{\circ} 19^{\prime} & 6 ! 34 & 2 & 3\end{array}$

Binaria.

$$
\begin{gathered}
\Sigma 2909=\zeta \text { Aquarii. Mag. 4.0, 4.r. } \\
\alpha=22^{\mathrm{h}} 23^{\mathrm{m}} \cdot 7 \quad \delta=-0^{\circ} 32^{\prime} .
\end{gathered}
$$

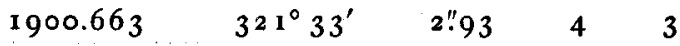

Binaria.

Barcelona, Observatorio Privado, 23 octubre 1900.
Epoca Angulo Distancia No. Peso

$$
\text { 2 2915. Mag. 8.5, 8.7. }
$$$$
\alpha=22^{\mathrm{h}} 27^{\mathrm{m}} \cdot 6 \quad \delta=+6^{\circ} 54^{\prime} \text {. }
$$

I $900.608 \quad$ I $50^{\circ} 34^{\prime}-11.98 \quad 2 \quad 3$

Movimiento retrógrado.

h $5367=\gamma$ Piscis Austrini. Mag. 4.5, 9.0. $\alpha=22^{\mathrm{h}} 47^{\mathrm{m}} \cdot \mathrm{o} \delta=-33^{\circ} 24^{\prime}$.

$\begin{array}{lllll}\text { 1 } 900.769 & 268^{\circ} 42^{\prime} & 3^{\prime \prime} 75 & 2 & 3\end{array}$

Lento movimiento retrógrado.

$$
\begin{gathered}
\text { Howe } 117=\beta 772=\delta \text { Piscis Austrini. } \\
\text { Mag. } 4.2, \text { 10.0. } \\
\alpha=22^{\mathrm{h}} 50^{\mathrm{m}} 4 \delta=-33^{\circ} 4^{\prime} . \\
1900.7^{6} 9 \quad 239^{\circ} 18^{\prime} \quad-\quad 2
\end{gathered}
$$
invariable.

La distancia es imposible de medir. Sensiblemente

$$
\begin{aligned}
& \beta 279=\omega^{2} \text { Aquarii. Mag. 5.0, I 1.0. } \\
& \alpha=23^{\mathrm{h}} 37^{\mathrm{m}} \cdot 5 \quad \delta=-15^{\circ} 6^{\prime} \text {. }
\end{aligned}
$$

La distancia es imposible medirla por la pequeñez de B.

h $5417=$ G. 26 Sculptoris. Mag. 6.0, 10.2.

$$
\alpha=23^{\mathrm{h}} 39^{\mathrm{m}} \cdot 3 \quad \delta=-26^{\circ} 48^{\prime} \text {. }
$$

$1900.75^{6} \quad 324^{\circ} 22^{\prime} \quad 7.8$ I $\quad 2 \quad$ I

Una sola medida de distancia. Lento movimiento.

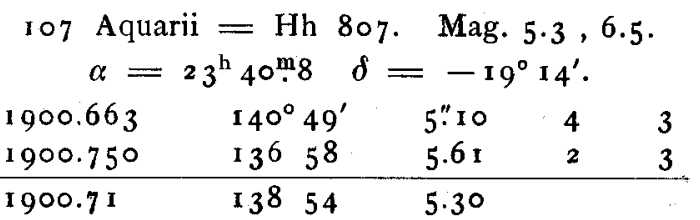

En la primera noche hay dos medidas de ángulo. Sensiblemente fija.

$$
\begin{aligned}
& 85 \text { Pegasi. Mag. 6.0, 9.0. } \\
& \alpha=23^{\mathrm{h}} 5^{6^{\mathrm{m}} .8} \delta=+26^{\circ} 34^{\prime} \text {. } \\
& \text { I } 900.682 \quad 340^{\circ} \text { I } 3^{\prime} \quad 35^{\prime \prime O I} \quad 2 \quad 3 \\
& \text { Rapido movimiento. }
\end{aligned}
$$

F. Comas Solá.

\title{
Pianeta (1) Cerere
}

osservato nell'opposizione r900 in Arcetri all'Equatoriale di Amici.*)

Obiettivo $284 \mathrm{~mm}$. Micrometro a lamine r 9.45 . Ingr. 124.

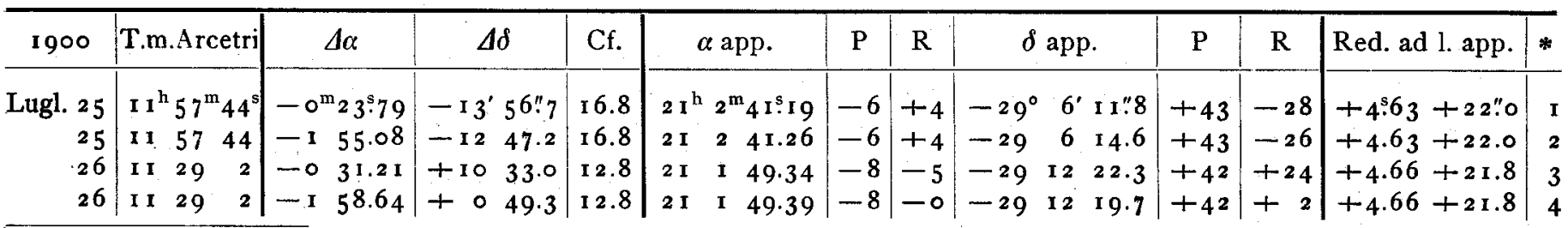

*) Nell' occasione che si pubblicano queste osservazioni si desidera ricordare che nel prossimo gennajo rgor si compie il centenario della famosa scoperta del Piazzi a Palermo (che fu fondamentale nella storia degli Asteroidi), la scoperta di Cerere. 


\begin{tabular}{|c|c|c|c|c|c|c|c|c|c|c|c|c|c|c|c|c|c|c|}
\hline 1900 & T.m.Arcetri & & $\Lambda \alpha$ & $\Delta$ & & Cf. & & $\alpha$ ap & pp. & $\mathrm{P}$ & $\mathrm{R}$ & $\delta a$ & app. & & $\mathrm{P}$ & $\mathbf{R}$ & Red. ad 1. app. & * \\
\hline 27 & $11^{h} 16^{\prime}$ & $-1^{n}$ & 66 & + & 30.8 & 6.8 & $2 \mathrm{I}^{\mathrm{h}}$ & $\mathrm{m}$ & 56.92 & -9 & -2 & $-29^{\circ}$ & & .77 & -42 & $\mid \begin{array}{ll}11 \\
1\end{array}$ & $-4^{5} 69+$ & \\
\hline 27 & $\begin{array}{lll}11 & 16 & 12\end{array}$ & -2 & 51.09 & 一 & 14.7 & 16.8 & $2 \mathrm{I}$ & 0 & .97 & -9 & +3 & -29 & I 8 & 23.9 & +42 & -12 & $-4.69+21.6$ & \\
\hline 28 & II 34 & +3 & 2.39 & +5 & 25.4 & 16.8 & $2 \mathrm{I}$ & $\circ$ & 3.03 & -6 & -2 & $-29=$ & 24 & .3 & +43 & +12 & $+4.71+21.4$ & \\
\hline 28 & $\begin{array}{ll}11 & 34\end{array}$ & $+I$ & 46.44 & +7 & 41.6 & 16.8 & 21 & 0 & 2.84 & -6 & -3 & -292 & 24 & 25.6 & +43 & +16 & $.71+$ & \\
\hline 29 & $\begin{array}{lll}11 & 10 & 18\end{array}$ & +2 & 9.46 & -0 & 22.8 & I 6.8 & 20 & 59 & 0.1 I & -8 & +2 & -293 & 30 & .5 & +42 & $-I$ & $.72+$ & \\
\hline 29 & $\begin{array}{lll}11 & 10 & 18\end{array}$ & +0 & 53.59 & +1 & 51.9 & 16.8 & 20 & 59 & 0.00 & -8 & $-\mathbf{I}$ & -293 & 30 & I $5 \cdot 3$ & +42 & +4 & $72+21.4$ & \\
\hline 30 & I I I 2 & +1 & 15.14 & -6 & 10.7 & 16.8 & 20 & 58 & 15.80 & -8 & +3 & -293 & 36 & 2.5 & +42 & -14 & $73+$ & \\
\hline 30 & I I I 2 & & - & -3 & 54.0 & -.8 & & - & & - & - & -293 & $3^{6}$ & 1.3 & 2 & -9 & $+4.73+$ & \\
\hline $3^{1}$ & $10 \quad 5429$ & +0 & 21.43 & - I I & $45 \cdot 5$ & $\mathrm{I} 6.8$ & 20 & 57 & 0 & -9 & +7 & -29 & $4 \mathrm{I}$ & $37 \cdot 3$ & +42 & -30 & $4.74+$ & \\
\hline 31 & 105429 & -0 & & -9 & 29.8 & 16.8 & 20 & 57 & 21.98 & -9 & +6 & -29 & 41 & .1 & +42 & -24 & $+4.74+$ & \\
\hline 1 & $\begin{array}{llll}1 & 1 & 2 & 1\end{array}$ & +2 & 19.43 & +4 & I 1.9 & 16.8 & 20 & 56 & 26.22 & -6 & $-\mathbf{I}$ & -29 & 47 & 20.3 & +43 & +9 & $.76+21.1$ & 7 \\
\hline 2 & I I 639 & +1 & 25.08 & $-\mathbf{I}$ & 10.4 & 16.8 & 20 & 55 & 31.90 & -7 & $+\mathbf{I}$ & -29 & 52 & 42.7 & +43 & -3 & $+4.79+21.0$ & 7 \\
\hline 3 & $103^{6} 3^{I}$ & -0 & 27.39 & +8 & 48.8 & r 4.8 & 20 & 54 & 38.34 & -9 & -6 & -29 & 57 & 53.0 & +42 & +24 & $+4.8 I+20.9$ & \\
\hline 3 & Io $3^{6} 3^{1}$ & -0 & $30.6 \mathrm{I}$ & +10 & $5 \cdot 7$ & I 4.8 & 20 & 54 & 38.22 & -9 & -7 & -29 & 57 & .9 & +42 & +27 & $+4.81+20.9$ & \\
\hline 7 & 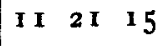 & +1 & $43 \cdot 4^{2}$ & +12 & 59.5 & I 6.8 & 20 & 50 & $57 \cdot 35$ & -3 & -2 & -30 & 17 & 50.4 & +43 & +28 & $+4.87+20.2$ & 10 \\
\hline 7 & 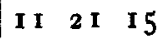 & +1 & I 2.57 & +12 & I I. I & r 6.8 & 20 & 50 & 57.46 & -3 & -2 & -30 & 17 & 49.2 & +43 & +26 & $+4.87+20.2$ & I I \\
\hline
\end{tabular}

$\mathrm{P}=$ Parallasse in centesimi di secondo di tempo per dissimo. - Agosto 2, 3. Sereno mediocre. - Agosto 7. l'ascensione retta, ed in decimi di secondo d'arco per la Splendidissimo, chiaro di luna prossima al Plenilunio. In declinazione.

$\mathrm{R}=$ Rifrazione nelle suddette unità.

Grand. media, $7 \cdot 4$, corrispondente alla data luglio $30^{\mathrm{d}} \cdot 74$.

Luglio 25, 26, 27. Sereno. - Luglio 28. Brutto sereno. - Luglio 29, 30, 31. Splendidissimo. - Agosto 1. Splendiquesta sera in cui per causa dello splendor di luna non si vedono le piccole stelle si giudica con sicurezza che Cerere splende assai più di * 10 (in Cord. DM. posta di $7 \cdot 8$ ), e che * 10 splende assai di più di * I I $\left(8 \mathrm{~m}_{3}\right)$ quindi si conclude esser Cerere quasi di $7^{\mathrm{m}}$ cioè più grande di quello che dà il Naut. Almanac $7 \cdot 8$.

Stelle di confronto.

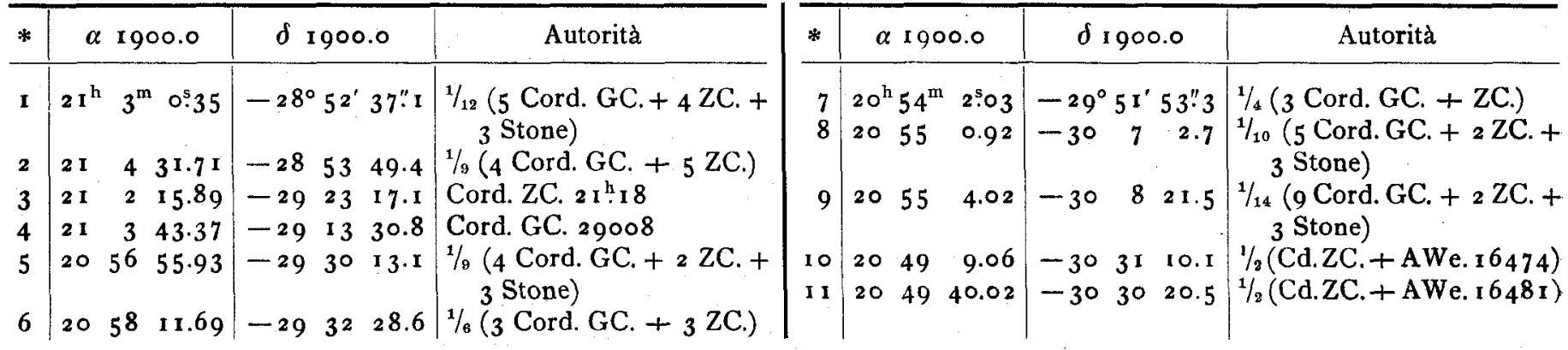

Confronto delle osservazioni coll' effemeride del Nautical Almanac I900 (O-C).

\begin{tabular}{r|c|c|r|r|r|r|r|r}
\hline \multicolumn{1}{c|}{1900} & $\Delta \alpha$ & $\Delta \delta$ & I900 & $\Delta \alpha$ & $\Delta \delta$ & I900 & $\Delta \alpha$ & $\Delta \delta$ \\
\hline Lugl. 25 & +2.02 & +5.1 & Lugl. 28 & +1.83 & +8.9 & Agos. I & +2.03 & +3.0 \\
25 & +2.09 & +2.5 & 29 & +2.12 & +6.5 & 2 & +1.86 & +3.5 \\
26 & +1.58 & +4.3 & 29 & +1.98 & +6.2 & 3 & +1.94 & +7.0 \\
26 & +1.68 & +4.7 & 30 & +2.07 & +4.9 & 3 & +1.81 & +5.4 \\
27 & +1.75 & +4.1 & 30 & - & +6.6 & 7 & +1.79 & +5.4 \\
27 & +1.85 & +2.6 & 31 & +2.18 & +4.7 & 7 & +1.90 & +6.4 \\
28 & +2.03 & +7.8 & 31 & +2.05 & +5.5 & & &
\end{tabular}

Questa serie di $\mathrm{O}-\mathrm{C}$ [osservazione meno effemeride] non presenta alcuna variazione proporzionale al tempo. Malgrado che le osservazioni abbiano per fondamento posizioni di stelle con differente grado di bontà tuttavia gli $\mathrm{O}-\mathrm{C}$ possono riguardarsi tutti di egual peso, eccettuati quelli di ascensione retta del 26 luglio a cui si puó dare il peso 1/2

perchè basati non solo su posizioni di stelle dedotte da un unico catalogo, ma anche su $\mathbf{2}_{2}$ confronti anziche su 16 , che è il numero normale adottato per un' osservazione tipo in circostanze pienamente favorevoli. Facendo dunque il medio di tutti gli $\mathrm{O}-\mathrm{C}$ si ottiene per la data media luglio $30^{\mathrm{d}} .74$ $\Delta a=+1.94 \quad \Delta \delta=+5 " 3$.

Arcetri-Firenze, I 900 Ottobre.

A. Abetti. 